\title{
Semantic-based Estimation of Term Informativeness
}

\author{
Kirill Kireyev \\ University of Colorado - Boulder \\ kireyev@colorado.edu
}

\begin{abstract}
The idea that some words carry more semantic content than others, has led to the notion of term specificity, or informativeness. Computational estimation of this quantity is important for various applications such as information retrieval. We propose a new method of computing term specificity, based on modeling the rate of learning of word meaning in Latent Semantic Analysis (LSA). We analyze the performance of this method both qualitatively and quantitatively and demonstrate that it shows excellent performance compared to existing methods on a broad range of tests. We also demonstrate how it can be used to improve existing applications in information retrieval and summarization.
\end{abstract}

\section{Introduction}

The idea that some words carry more semantic content than others has been occurring in various literature in linguistics, psychology and computer science for some time. The intuitive notion of specificity has long existed before it was formalized; consider, for example, the distinction between the more general word "beverage" and more specific terms "tea", "coffee" and "cocoa" made by Spärck-Jones (1973). Another informal mention of specificity is mentioned by Gorman (1961):

A word may be "abstract" and either general or specific, or "concrete" and either general or specific.

where it is contrasted with another psycholinguistic property of concreteness, which is generally defined as "the extent to which the word's referent can be touched or felt" (Reilly et al., 2007).

The field of information retrieval has attracted greater attention to the computational estimation and applications of term specificity. It has been noted that words with higher specificity, or information content, deserve to be weighted more heavily when matching documents with queries, since these words play a greater importance in characterizing what a query or a document is about. By contrast, stopwords, words that contribute the least amount of semantic content, are often downweighted or removed altogether (see (Lo et al., 2005), for example).

In addition to IR, term specificity, or informativeness, has been shown useful in other applications, such as Named Entity Tagging (Rennie et al., 2005), creating back-of-the-book glossaries (Csomai et al., 2007), and extractive summarization (Kireyev, 2008).

A related notion of communication density has been introduced by Gorman et al. (2003) in team communication analysis, to measure the extent to which a team conveys information in a concise manner, or, in other words, the rate of meaningful discourse, defined by the ratio of meaningfulness to number of words spoken. The meaningfulness described here should not be confused with psycholinguistic quality of meaningfulness as described by Toglia and Battig (1978), which is the degree to which a word is associated with other words.

In this paper we consider the terms specificity, informativeness and information content of words to mean the same thing. A precise formulation or analysis of important qualitative characteristics of these concepts has not been performed in previous literature; we hope to make some progress in that direction in this paper.

Our main goal is to introduce a new method of computing word specificity based on the rate and strength of semantic associations between words, as modeled by Latent Semantic Analysis (LSA).

\section{Previous Approaches}

To date, most of the known approaches to estimating term informativeness have relied on frequency-based methods. 
A very basic, yet surprisingly effective approach to measuring term informativeness is its frequency of occurrence in a large representative corpus of language. Spärck Jones (1973) defines $I D F$ or inverse document frequency, which is determined by the probability of occurrence of documents containing a particular word:

$$
I D F(w)=-\log _{2}\left(d f_{w} / D\right)
$$

where $D$ is the total number of documents in the corpus. The assumption behind it is that low frequency words tend to be rich in content, and vice versa.

Church and Gale (1995) correctly note that this measure is fundamentally different from collection frequency $f_{w}$, (the total number of times the word type occurs in the corpus) or its transformations, despite the fact that the two measures appear highly correlated. In fact, what is particularly of interest are the words for which these two quantities deviate the most. This happens most dramatically for most informative, or content words, such as "boycott" (Church, 1995a). These words happen to exhibit "bursty" behavior, where they tend to appear multiple times but in fewer documents, thus having $f_{w}>d f_{w}$. In contrast, less content-loaded words like "somewhat" tend to occur on average once in documents, and thus have similar values for collection and document frequencies $\left(f_{w} \approx d f_{w}\right)$. As a result, more informative words can be less accurately estimated by the Poisson distribution, which is based on the simplistic assumption that the expected number of occurrences of word in a document can be estimated by its total number of occurrences in the corpus.

Most prominent statistical measures of term informativeness rely on quantifying this notion of deviation from the Poisson distribution. If the mean expected word rate is:

$$
\overline{t_{w}}=\frac{f_{w}}{D}
$$

then the variance metric can be defined as:

$$
\operatorname{variance}(w)=\frac{1}{D-1} \sum_{d=1}^{D}\left(t_{d w}-\overline{t_{w}}\right)^{2}
$$

where $t_{d w}$ is the actual number of occurrences of term $w$ in document $d$. The idea is that a higher variance would indicate greater deviation from ex- pected frequency of occurrence in a document, which is assumed to be higher for informative words.

Another measure, suggested by Church and Gale (1995a) is burstiness which attempts to compare collection frequency and document frequency directly:

$$
\operatorname{burstiness}(w)=\frac{\overline{t_{w}}}{d f_{w} / D}=\frac{f_{w}}{d f_{w}}
$$

Church and Gale also noted that nearly all words have IDF scores that are larger than what one would expect according to an independence-based model such as the Poisson. They note that interesting or informative words tend to have the largest deviations from what would be expected. They thus introduce the notion of residual IDF which measures exactly this deviation:

$$
\text { residualIDF }(w)=I D F(w)+\log _{2}\left(1-e^{-\bar{t}}\right)
$$

Papineni (2001) introduces the notion of gain:

$$
\operatorname{gain}(w)=\frac{d f_{w}}{D}\left(\frac{d f_{w}}{D}-1-\log \left(\frac{d f_{w}}{D}\right)\right)
$$

This measure tends to give low weights to very high- and very low- frequency words.

Most closely related to our work is the notion of meaningfulness in (Gorman et al 2003), computed as the LSA vector length. We will discuss it further in the subsequent sections, and show that a small but crucial modification to this quantity gives the best results.

\section{Using Latent Semantic Analysis for Ap- proximating Term Informativeness}

\subsection{Latent Semantic Analysis}

Latent Semantic Analysis (LSA) is a language model that represents semantic word meaning as vectors in high-dimensional space. Word vectors are positioned in such a way that semantically-related words vectors point in similar directions or have a smaller angle / higher cosine between them. The representation is derived in an unsupervised manner, by observing occurrence patterns of words in a large corpus of natural language documents. Singular Value Decomposition on the matrix of word/document occurrence counts is used to derive the optimal set of dimensions of the space in which 
all of the words can be represented as vectors. The number of dimensions is then artificially reduced to a smaller number (typically around 300) of most important dimensions, which has the effect of smoothing out incidental relationships and preserving significant ones between words.

The resulting geometric space allows for straightforward representation of meaning of words and/or documents; the latter are simply a weighted geometric composition of constituent word vectors. Similarity in meaning between a pair of words or documents can be obtained by computing the cosine between their corresponding vectors. For details of LSA, please see (Landauer et al., 2007), and others

\subsection{LSA Term Vector Length}

Most of the LSA applications focus on comparing semantic similarity between words and/or text, using the cosine measure of the angle between the corresponding vectors. There is, however, another significant characteristic of LSA word vectors besides their direction in space; it is their vector length. The vector length for words differs significantly, as is shown in Table 1.

\begin{tabular}{|l|r|r|}
\hline \multicolumn{1}{|c|}{ Word } & $\boldsymbol{d f}_{\boldsymbol{w}}$ & \multicolumn{1}{c|}{ Vector Length } \\
\hline dog & 1365 & 1.3144 \\
\hline green & 2067 & 0.7125 \\
\hline run & 2721 & 0.4788 \\
\hline puppy & 127 & 0.2648 \\
\hline electron & 264 & 0.9009 \\
\hline the & 44474 & 0.0098 \\
\hline
\end{tabular}

Table 1: LSA vector length for some of the words in TASA corpus.

The vector length plays a very important role in many LSA calculations, in particular - in giving relative weights to the word vectors that constitute a particular text passage.

What causes differences in vector lengths? They are based roughly on how much information LSA learns about a word based on its patterns of occurrence in the corpus. Kintsch (2001) writes:

Intuitively, the vector length tells us how much information LSA has about this vector. [...] Words that LSA knows a lot about (because they appear frequently in the training corpus[...]) have greater vector lengths than words LSA does not know well. Function words that are used frequently in many different contexts have low vector lengths -- LSA knows noth- ing about them and cannot tell them apart since they appear in all contexts.

Essentially, there are two factors that affect vector length: (1) number of occurrences and (2) the consistency of contexts in which the word occurs.

\subsection{Deriving Specificity from Vector Length}

Based on the observations above we propose a new metric of term informativeness, or specificity, which we call LSAspec, which is simply the ratio of LSA vector length to the number of documents in the LSA training corpus that contain a particular word:

$$
\operatorname{LSAspec}(w)=\|\vec{w}\| / d f_{w}
$$

The value can be interpreted as the rate of vector length growth. We argue that more specific, or informative, words have the greatest rate of vector length growth; LSA learns about their meaning faster, with relatively fewer exposures. To illustrate this concept, let's look at a few examples, that were obtained by controlling the number of occurrences of a particular word in the LSA training corpus. The base corpus was obtained using the 44000-passage TASA corpus with all passages containing the three words below initially removed. Each data point on the graph reflects the vector length of a particular word, after training LSA on the base corpus plus the specified number of passages containing a particular word added back. Highly specific words like "cellulose" gain vector length quite quickly compared to a low-specificity word like "dismay".

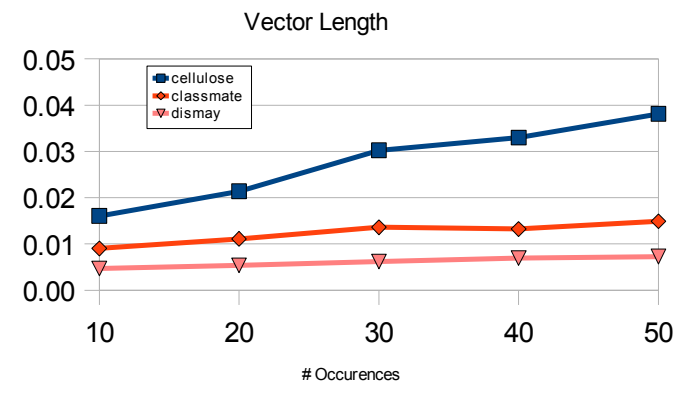

Illustration 1: Vector lengths for some words vs the number of documents containing those words.

\section{Comparison of Specificity Metrics}

Past attempts to examine the merits of various existing term informativeness estimation methods in the literature thus far has largely involved em- 
pirical summative evaluations as part of information retrieval or named entity tagging systems (Rennie et al., 2005). Here, we provide some measures which hopefully provide more illuminating insights into the various methods.

In all of the tests below we derived the metrics (including the LSA space for LSAspec) from the same corpus - MetaMetrics 2002 corpus, composed of $\sim 188 \mathrm{k}$ passages mostly used in educational texts. No stemming or stopword removal of any kind was performed. All word types were converted to lowercase. We computed the specificity score for each of the 174,374 most frequent words in the corpus using each of the metrics described above: LSAspec, IDF, residualIDF, burstiness, gain and variance.

\subsection{Correlation with Number of Senses}

Intuitively, one would expect more specific words to have more precise meaning, and therefore, generally fewer senses. For example, "electron" is a specific physics term that has only one sense, whereas "run" has a very general meaning, and thus has over 50 senses in the WordNet database (Miller et al., 1990). There are many exceptions to this, of course, but overall, one would expect a negative correlation between specificity and number of senses.

In this test, we measure the correlation between the specificity score of a word by various methods and its number of senses in WordNet version 3.0. A total of 75,978 words were considered. We use Spearman correlation coefficient, since the relationships are likely to be non-linear.

\begin{tabular}{|l|r|l|r|}
\hline \multicolumn{1}{|c|}{ Metric } & \multicolumn{1}{c|}{ Corr } & \multicolumn{1}{c|}{ Metric } & \multicolumn{1}{c|}{ Corr } \\
\hline LSAspec & $\mathbf{- 0 . 4 6}$ & burstiness & -0.02 \\
\hline IDF & -0.44 & variance & 0.40 \\
residualIDF & -0.03 & gain & 0.44 \\
\hline
\end{tabular}

Table 2: Correlation of specificity metrics with number of senses in WordNet

LSAspec gives the highest negative correlation with number of WordNet senses.

\subsection{Correlation with Hypernymy}

WordNet organizes concepts into a hypernymy tree, where each parent node is a hypernym of the child node below it. For example:

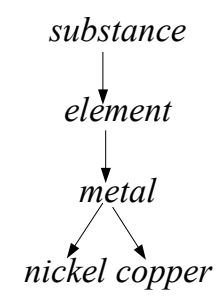

In general one would expect that for each pair of child-parent pairs in the hypernym tree, the child will have greater specificity than the parent ${ }^{1}$. We examined of a total of 14451 of such hypernym word pairs and computed how often the child's informativeness score, according to each of the measures, is greater than its parent's (its hypernym's) score.

\begin{tabular}{|l|r|l|r|}
\hline \multicolumn{1}{|c|}{ Metric } & Percent & \multicolumn{1}{c|}{ Metric } & \multicolumn{1}{c|}{ Percent } \\
\hline IDF & $\mathbf{8 8 . 8 \%}$ & burstiness & $47.2 \%$ \\
\hline LSAspec & $87.7 \%$ & variance & $13.4 \%$ \\
residualIDF & $48.8 \%$ & gain & $11.1 \%$ \\
\hline
\end{tabular}

Table 3: Percentage of the time specificity of child exceeds that of its hypernym in WordNet

\subsection{Writing Styles and Levels}

One may expect that the specificity of words on average would change with texts that are known to be of different writing styles and difficulty level. To test this hypotheses we extracted texts from the TASA collection of educational materials. The texts are annotated with genre ("Science", "Social Studies" or "Language Arts"), and difficulty level on the DRP readability scale (Koslin et al., 1987). Intuitively, one would expect to see two patterns among these texts:

(1) The specificity of words would generally increase with increasing level of difficulty of texts.

(2) Informative (Science) texts should have more specific terms than narrative (Language Arts) texts; with Social Studies somewhere in between (McCarthy et al., 2006).

We extracted 100 text passages for each combination of style ("Science", "Social Studies", "Language Arts") and DRP difficulty level (50, 55, $60,65,70)^{2}$, thus resulting in 15 batches of 100 passages. For each passage we computed the median specificity measure of each unique word type in

\footnotetext{
In practice this is more difficult to determine, since some WordNet entries are actually phrases, rather than words (e.g. "tulip" $\leftarrow$ "liliaceous plant" $\leftarrow \ldots \leftarrow$ "plant"). In such cases we search up the tree until we stumble upon a node where the entry (or one of the entries) is a single word.

2 DRP level of 50 roughly corresponds to the beginning of $6^{\text {th }}$ grade in US schools, 70 corresponds to end of $10^{\text {th }}$ grade.
} 
the passage, and averaged these values over 100 passages of each batch. Table 4 shows the results.

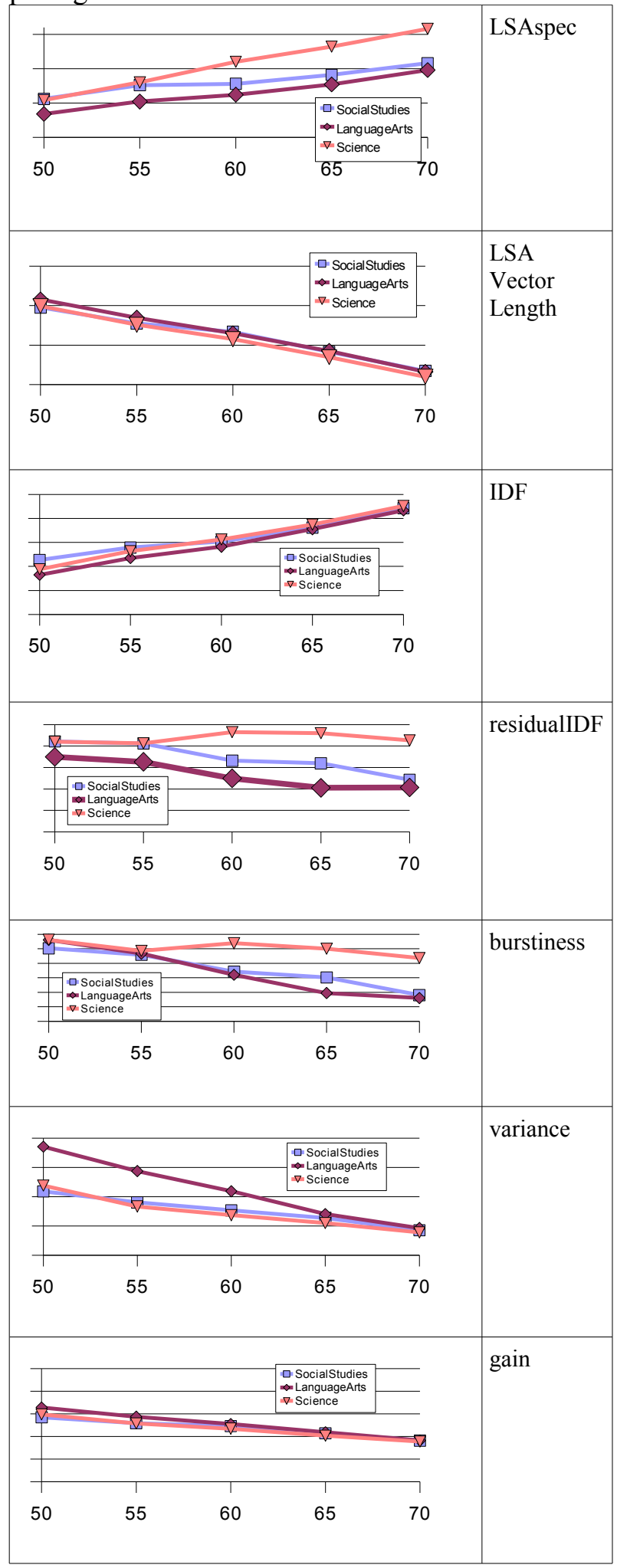

Table 4: Average median specificity scores for texts of different genres and DRP levels.
Note that the absolute values for a particular batch of texts are not important in this case; it's the relative differences between batches of different styles and difficulty levels that are of interest. Of all the measures, only LSAspec appears to exhibit the two characteristics described above (increasing with text difficulty, and separating the three genres in the expected way). The metrics residualIDF and burstiness also appear to separate the genres as expected, but they do not increase with text difficulty.

It is also evident that LSA Vector Length alone does not serve as a good measure of informativeness, contrary to its use as such in (Gorman et al., 2003). In fact, it shows the most dramatic and reliable inverse relationship with text difficulty. This is likely due to the fact that texts of lower difficulty use common (easier) words more often; these words tend to have longer LSA vector lengths.

\subsection{Back-of-the-Book Glossary}

Educational textbooks typically have a glossary (index) at the end which lists important terms or concepts mentioned in the book. One would expect these terms to have greater informativeness compared to other words in the textbook. This was a crucial assumption used by Csomai and Mihalcea (2007), who used informativeness (as measured by $I D F$ and other metrics) as one of the main features used to automatically generate glossaries from textbooks.

We can use existing textbooks and their glossaries to validate this assumptions, by observing the extent to which the words in the glossary are ranked higher by different specificity metrics compared to other words. Note that the goal here is not to actually achieve optimal performance in automatically finding glossary words; for this reason we do not use recall/precision- based evaluation or rely on additional features such as term frequency (or the popular $t f_{w} \cdot i d f_{w}$ measure). Rather the goal is to simply see how much the glossary words exhibit the property (informativeness) that we are trying to compute with various methods.

We obtained a collection of textbook chapters (middle-school level material from Prentice Hall Publishing) and their corresponding glossaries, in two different genres: 8 on World Studies (e.g. "Africa", "Medieval Times") and 13 on Science (e.g. "Structure of Animals", "Electricity"). Each 
chapter was converted into text and a list of unique words was extracted.

For each of the specificity metrics, we compute how well it predicts glossary words:

1. Compute the specificity of each word in a chapter, according to the metric.

2. Order all the words in decreasing order of specificity.

3. Compute the median percentile rank (position) in the list above of all single-word entries in the glossary (top word has the rank of 0 ; bottom has a rank of 100).

If a specificity metric predicts the glossary words well, we would expect the average rank to be low; i.e. glossary words would be near the top of the specificity-ordered list.

\begin{tabular}{|l|r|r|}
\hline \multicolumn{1}{|c|}{ Metric } & \multicolumn{1}{|c|}{ Word Studies } \\
& $\begin{array}{l}(\sim 9000 \text { total wds } / \mathrm{ch} \\
\sim 260 \text { gloss wds } / \mathrm{ch})\end{array}$ & $\begin{array}{c}\text { Science } \\
(\sim 1000 \text { total wds } / \mathrm{ch} \\
\sim 20 \text { gloss wds } / \mathrm{ch})\end{array}$ \\
\hline LSAspec & $\mathbf{0 . 2 1}$ & $\mathbf{0 . 1 0}$ \\
\hline residualIDF & 0.21 & 0.11 \\
\hline burstiness & 0.21 & 0.12 \\
\hline IDF & 0.29 & 0.16 \\
\hline variance & 0.49 & 0.64 \\
\hline gain & 0.51 & 0.68 \\
\hline
\end{tabular}

Table 5: Average median rank of glossary words among all other words in textbook by specificity.

LSAspec shows the lowest median percentile for both genres of books.

\subsection{Qualitative Analysis}

It is useful to inspect the significant differences between the word rankings by different methods, to see if some notable patterns emerge. We can find words on which the methods disagree most dramatically by observing which of them have the most significant differences of position (0-100) in the word lists ranked by different specificity metrics. To avoid dealing with overly-rare words, we restrict our attention to the 23,000 most frequent words in the corpus.

Let's first compare LSAspec and residualIDF. From the list of 100 words with the most extreme disagreements, we select some examples that have high rank for LSAspec (and low for residuaIDF) and vice-versa. From Table 6 we can see that residualIDF misses some term words (such as "chromatin") which LSAspec correctly rates as highlyspecific words. Conversely, residualIDF, incor- rectly ranks common words like "her" and "water" as highly-specific. The reason for this behavior is that words like "chromatin" happen to occur only once per document in the texts they are mentioned (e.g. $d f_{\text {cromatin }}=t f_{\text {chromatin }}=7$ ), whereas "her" and "school" tend to occur frequently per document. In real applications "her" will probably be discarded using stopword lists, but "school" will probably not.

\begin{tabular}{|l|r|r|}
\hline \multicolumn{1}{|c|}{ Word } & LSAspec & \multicolumn{2}{c|}{ residualIDF } \\
\hline oviducts & 0.5 & 98.8 \\
cuspids & 0.6 & 98.8 \\
\hline chromatin & 0.7 & 98.7 \\
disassembly & 0.7 & 98.7 \\
\hline her & 99.9 & 1.5 \\
\hline my & 99.9 & 3.5 \\
water & 97.5 & 5.1 \\
\hline school & 97.8 & 10.3 \\
\hline
\end{tabular}

Table 6: Words ranked most differently by LSAspec and residualIDF

Comparing LSAspec and burstiness we see almost the same pattern, which is not surprising, since burstiness and residualIDF work from the same assumptions that content words tend to occur multiple times but in fewer documents.

The table below lists examples of most notable differences between LSAspec and IDF.

\begin{tabular}{|l|r|r|}
\hline \multicolumn{1}{|c|}{ Word } & LSAspec & IDF \\
\hline billy & 10.3 & 93.5 \\
\hline jack & 15.0 & 95.9 \\
melody & 4.1 & 83.8 \\
\hline cells & 10.8 & 86.3 \\
\hline inducing & 34.0 & 9.8 \\
vagueness & 32.5 & 9.6 \\
initiating & 31.5 & 8.7 \\
\hline apathetic & 32.3 & 9.8 \\
\hline
\end{tabular}

Table 7: Words ranked most differently by LSAspec and $I D F$ and their percentiles

There is a large disagreement between rankings of common proper names (e.g. "jack"). It is not clear what the correct answer for these should be, although Rennie \& Jaakkola (2005) use informativeness for named entity detection, assuming that proper names should have high specificity. Common but important words like "melody" and "cells" are considered low-specificity by IDF. By 
contrast, rare but vague words like "inducing" or "vagueness" are improperly given a high specificity ranking.

\section{Applications in LSA}

Having demonstrated that our word specificity metric performs well with regards to some natural linguistic phenomena, we can now show that it can be used successfully as part of existing NLP technologies. Here we will focus particularly on applications within Latent Semantic Analysis (LSA), although it is highly likely that this specificity metric can be used successfully in other places as well.

We will demonstrate that LSAspec shows better results that the conventional term weighting scheme in LSA. It is also important to note that although LSAspec is derived using LSA, it is in fact logically independent from the term weighting mechanism used by LSA; other metrics (such as the ones described above) could also be potentially used for LSA term weighting.

In order to represent the meaning of text in LSA, one typically computes the document vector of the text by geometric addition of word vectors for each of the constituent words:

$$
\vec{V}_{d}=\sum_{w \in d} a_{w} * \log \left(1+t_{d w}\right) * \vec{v}_{w}
$$

where $a_{w}$ is the log-entropy weight of the word $w$, typically set to $t f_{w} \cdot i d f_{w}$ (or some variation thereof), $t_{d w}$ is the number of occurrences of the word $w$ in the document, and $v_{w}$ is the vector of the word. Implicit in $v_{w}$ is its geometric length, which tends to be much greater for frequently-used words (unless they are extremely vague). It is tempered somewhat by $a_{w}$ which is higher for content words, but perhaps not effectively enough, as the subsequent tests will show. McNamara et al. (2007) experimented with changing the weighting scheme, mainly focusing on prioritizing rare vs. frequent words, and has shown significant differences in short-sentence comparison results.

In the sections below we compare the original LSA weighting scheme with our new scheme based on LSAspec:

$$
\vec{V}_{d}=\sum_{w \in d} \operatorname{LSAspec}(w) * \log \left(1+t_{d w}\right) * \frac{\vec{v}_{w}}{\left\|\vec{v}_{w}\right\|}
$$

In other words, we replace the weight parameter $a_{w}$ and the implicit weight contained in the length of each word vector (by normalizing it) with the specificity value of $L$ SAspec.

We show that the resulting term weighting scheme improves performance in three important applications: information retrieval, gisting and short-sentence comparison.

\subsection{Information Retrieval}

LSA was first introduced as Latent Semantic Indexing (Deerwester et al, 1990), designed for the goal of more effective information retrieval by representing both documents and queries as vectors in a common latent semantic space.

In this IR context, the type of term weighting used to compose document and query vectors plays an important role. We show that using our LSAspec-based term weighting gives superior performance to the traditional weighting scheme described in the previous section.

We used the SMART Time ${ }^{3}$ dataset, a collection of 425 documents and 83 queries related to Time magazine news articles. For this task only, we used a LSA space that was built using the AQUAINT-2 corpus $^{4}$, a large collection $(\sim 40,000)$ of news articles from prominent newspapers such as the New York Times. The variable parameter in the LSA IR models was the cosine threshold between the document and the query, which was varied between 0 and 1

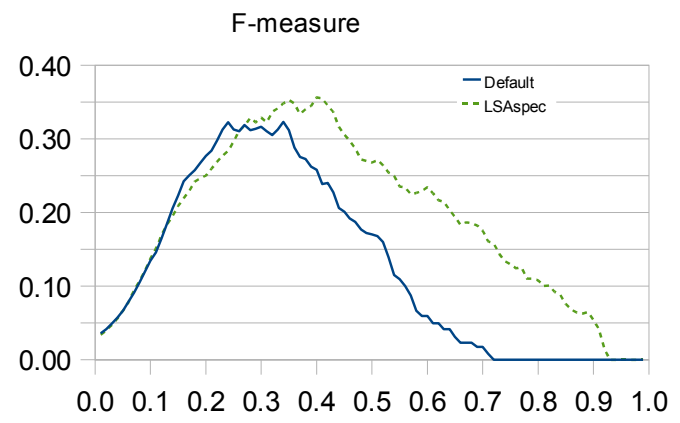

Figure 2: The performance of default LSA and LSA+LSASpec on SMART IR dataset.

Figure 1 shows the performance of the original LSA and LSA with LSAspec ${ }^{5}$ term weighting method, in terms of the F-measure, which is the harmonic mean of precision and recall; a higher value means better performance. The abscissa in

ftp://ftp.cs.cornell.edu/pub/smart/time/

TREC conference: http://trec.nist.gov/

LSAspec measure was the same as before, derived from LSA built on MetaMetrics corpus. 
the graph is the value of the threshold cosine parameter. The LSAspec term weighting outperforms the original term weighting.

\subsection{Sentence Similarity}

Here we analyze performance of the two LSA term weighting methods on automated sentence similarity comparisons. Although LSA works best on units of text of paragraph-size or larger, it can work reasonably well on sentence-length units.

We use the dataset reported by McNamara (2007), where the authors collected a set of sentence pairs from several books. A total of 96 sentence pairs was provided, consisting of a combination of subsequent sentences in the book (16), nonadjacent sentences in the same book (16), sentences from two different books (48), and sentences where one is a manually-created paraphrase of one another (16). The standard of reference for this task is human similarity ratings of these sentences within each pair, reported on a Likert scale between 6 (most similar) and 1 (completely dissimilar). Here we report correlations between human rating and LSA similarity with the two term weighting metrics.

\begin{tabular}{|ll|ll|}
\hline Original LSA: & 0.66 & LSA + LSAspec: & 0.85 \\
\hline
\end{tabular}

Using LSAspec term weighting gives better performance compared to the original LSA term weighting scheme.

\subsection{Gisting (Very Short Summarization)}

The ability to represent documents and words in a common geometric space allows LSA to easily compute the gist of a document by finding the word (or sentence) whose vector is most similar by cosine metric to the document vector. This word can be interpreted as the most representative of the cumulative meaning of the document; it can also be thought as a one-word summary of the document. Gisting is discussed from a psychological perspective by Kintsch (2002).

Once again, the choice of term weighting mechanism can make a significant difference in how the overall document vector is constructed. Here, we compare the original weighting scheme and LSAspec in the performance on gisting. To perform this evaluation, we selected 46 well-written Wikipedia ${ }^{6}$ articles in various categories: Sports, Animals, Countries, Sciences, Religions, Diseases. The original single-word Wikipedia title of each of the articles can be thought as the optimal one-word gist of the article, thus serving as a reference answer in evaluation. A perfect gisting performance by the model would always select the original title as the closest word to the meaning of the document. We also measure the position of the original title in the list of all words in the article ranked by their similarity to the document vector, and ranging from 0 (original title picked as top word) and 1. Table 10 shows a few examples of both the top word and rank of the title, as well as the overall mean rank of all 46 articles.

\begin{tabular}{|l|l|l|l|l|}
\hline \multirow{2}{*}{ Title } & \multicolumn{2}{c|}{ Orig LSA } & \multicolumn{2}{c|}{ LSA + LSAspec } \\
\cline { 2 - 5 } & top word & rank & \multicolumn{1}{c|}{ top word } & rank \\
\hline Skiing & skiing & 0.0000 & skiing & 0.0000 \\
Thailand & buddhism & 0.0189 & thailand & 0.0000 \\
\hline Sociology & sociologists & 0.0012 & sociology & 0.0000 \\
Pneumonia & infections & 0.0064 & infections & 0.0092 \\
\hline Mean rank (all 46 articles) & $\mathbf{0 . 0 1 9 1}$ & & $\mathbf{0 . 0 0 6 1}$ \\
\hline St. dev. of rank & $\mathbf{0 . 0 8 4 7}$ & & $\mathbf{0 . 0 1 3 3}$ \\
\hline
\end{tabular}

Table 8: Examples of gisting (picking most representative word for text) in with and without LSASpec in LSA

Using LSAspec noticeably improves gisting performance, compared to the original LSA term weighting method, as is evidenced by much lower mean rank of the original title.

\section{Conclusion}

We have introduced a new method of measuring word informativeness. The method gives good results modeling some real linguistic phenomena, and improves LSA applications.

We attempted to look more deeply at the relevant characteristics of word specificity (such as correlation with number of senses). Our method seems to correspond with intuition on emulating a wide range of these characteristics. It also avoids a lot of pitfalls of existing methods that are based purely on frequency statistics, such as unduly prioritizing rare but vague words.

Further research should examine the stability of this method (compared to others) with regards to variation/size of the training corpus. It should also analyze application of the method in other natural language tasks. Lastly, it should be correlated with human judgments, similar to other psycholinguistic properties.

6 http://en.wikipedia.org, circa May 2008. 


\section{References}

Kenneth W. Church and William A. Gale. 1995. Poisson mixtures. Journal of Natural Language Engineering, 1995

Kenneth W. Church and William A. Gale. 1995a. Inverse document frequency (IDF): A measure of deviation from Poisson. In Proceedings of the Third Workshop on Very Large Corpora, pp 121-130, 1995.

András Csomai and Rada Mihalcea. 2007. Investigations in Unsupervised Back-of-the-Book Indexing. In Proceedings of the Florida Artificial Intelligence Research Society, Key West.

Scott Deerwester, Susan T. Dumais, George W. Furnas and Thomas K. Landauer. 1990. Indexing by Latent Semantic Analysis. Journal of the American Society for Information Science, 41.

Aloysia M. Gorman. 1961. Recognition Memory for Nouns as a Function of Abstractness and Frequency. Journal of Experimental Psychology. Vol. 61, No. 1.

Jamie C. Gorman, Peter W. Foltz, Preston A. Kiekel and Melanie J. Martin. 2003. Evaluation of Latent Semantic Analysis-based Measures of Team Communication Content. Proceedings of the Human Factors and Ergonomics Society, 47th Annual Meeting, pp 424-428.

Walter Kintsch. 2002. On the notions of theme and topic in psychological process models of text comprehension. In M. Louwerse \& W. van Peer (Eds.) Thematics : Interdisciplinary Studies, Amsterdam, Benjamins, pp 157-170.

Walter Kintsch. 2001. Predication. Journal of Cognitive Science, 25.

Kirill Kireyev. 2008. Using Latent Semantic Analysis for Extractive Summarization. Proceedings of Text Analysis Conference, 2008.

B. L. Koslin, S. Zeno, and S. Koslin. 1987. The DRP: An Effective Measure in Reading. New York College Entrance Examination Board.

Thomas K Landauer and Susan Dumais. 1997. A solution to Plato's problem: The Latent Semantic nalysis theory of the acquisition, induction, and representation of knowledge. Psychological Review, 104, pp 211-240.

Thomas K Landauer, Danielle S. McNamara, Simon Dennis, and Walter Kintsch. 2007. Handbook of Latent Semantic Analysis Lawrence Erlbaum.
Rachel TszWai Lo, Ben He, and Iadh Ounis. 2005. Automatically Building a Stopword List for an Information Retrieval System. 5th Dutch-Belgium Information Retrieval Workshop (DIR). 2005.

Philip M. McCarthy, Arthur C. Graesser, Danielle S. McNamara. 2006. Distinguishing Genre Using CohMetrix Indices of Cohesion. 16th Annual Meeting of the Society for Text and Discourse, Minneapolis, $M N, 2006$.

Danielle S. McNamara, Zhiqiang Cai, and MaxM. Louwerse. 2007. Optimizing LSA Measures of Cohesion. Handbook of Latent Semantic Analysis . Mahwah, NJ: Erlbaum. ch 19, pp 379-399.

George A. Miller, Richard Beckwith, Christiane Fellbaum, Derek Gross and Katherine Miller. 1990. WordNet: An on-line lexical database. International Journal of Lexicography, 3 (4), 1990.

Kishore Papineni. 2001. Why inverse document frequency. In Proceedings of the NAACL, 2001

Jamie Reilly and Jacob Kean. 2007. Formal Distinctiveness Of High- and Low- Imageability Nouns: Analyses and Theoretical Implications. Cognitive Science, 31.

Jason D. M. Rennie and Tommi Jaakkola. 2005. Using Term Informativeness for Named Entity Detection. Proceedings of ACM SIGIR 2005.

Karen Spärck-Jones. 1973. "A Statistical Interpretation of Term Specificity and its Application in Retrieval," Journal of Documentation, 28:1.

Michael P. Toglia and William R. Battig. 1978. Handbook of semantic word norms. Hillsdale, NJ: Lawrence Erlbaum Associates. 